\title{
Omotic lexicon in its Afro-Asiatic setting III: Omotic *p-
}

\begin{abstract}
The present paper is published as part of a set of papers whose goal is to identify the AfroAsiatic heritage in the Omotic lexicon according to initial consonants. The current installment deals with instances of Omotic ${ }^{*} p$ - and ${ }^{*} p^{h}$ - that may be traced back to Proto-AfroAsiatic $*$ - and ${ }^{*} f$-. Altogether, 28 etymologies are discussed under this section.

Keywords: Afro-Asiatic languages, Omotic languages, comparative phonology, historical reconstruction.
\end{abstract}

We owe much to H. C. Fleming, M. L. Bender, and M. Lamberti for their pioneering studies in Omotic lexical comparison and reconstruction. The latter two authors even managed to come up with a few monographs on the subject (Bender 1975, 1999, 2003, Lamberti 1993, Lamberti and Sottile 1997). Still, this language branch arguably represents the least cultivated field within the immense Afro-Asiatic domain from the viewpoint of systematic etymological elaboration of the inherited Afro-Asiatic lexical treasures. To the best of my knowledge, the only special study devoted to a systematic treatment of Omotic/Afro-Asiatic matches is the unpublished paper presented by V. Blažek at the $2^{\text {nd }}$ International Symposium on Cushitic and Omotic Languages (Torino, November 1989).

This kind of research has already brought considerable progress in other branches of the vast Afro-Asiatic language macrofamily (Semitic, Egyptian, Berber, Cushitic, Chadic). The abundant results in Semito-Egyptian linguistic comparison are well-known and are due to some two centuries' efforts (cf. EDE I 1-8). The Afro-Asiatic background of the Berber root inventory has been investigated in special studies by O. Rössler (1952, 1964), A. Militarev (1991), and most recently by myself in a series of papers entitled 'Some Berber etymologies' (Takács 2011 with a list of previous parts). Materials for an etymological dictionary of Bedawye (North Cushitic) have been collected and partially published by V. Blažek (1990 MS, 1994 MS, 2000 MS, 2003, 2006), whereas D. Appleyard (2006) has most recently concluded his Agaw (Central Cushitic) comparative lexicon. A. Dolgopolsky examined in 1983 the Semitic matches of East Cushitic roots, in 1988 the word-initial laryngeals and pharyngeals in Semitic vs. East Cushitic, and, in 1987, the South Cushitic laterals as compared to Semitic. Regular phonological and lexical correspondences between South Cushitic and Afro-Asiatic have been established by myself (Takács 1999, 2000, 2010). Numerous works by H. Jungraithmayr (e.g., JI 1994 I), O. Stolbova (e.g., 1996 or CLD I-III), and myself (e.g., in my diverse series like 'Angas-Sura etymologies', the one on North

${ }^{1}$ G. TAKÁCs. Angas-Sura Etymologies I = Lingua Posnaniensis 46 (2004), 131-144. Angas-Sura Etymologies II = Rocznik Orientalistyczny 57/1 (2004), 55-68. Angas-Sura Etymologies III = Lingua Posnaniensis 48 (2006), 121-138. Angas-Sura Etymologies IV: *f- = Folia Orientalia 47/2 (2011), 273-289. Angas-Sura Etymologies V: ${ }^{*} \mathrm{~m}-=$ Cahiers Caribéens d'Égyptologie 13-14 (2010), 137-142.

Journal of Language Relationship • Вопросы языкового родства • 8 (2012) • Pp. 103-116 • Takács G., 2012 
Bauchi $^{2}$ or the one on 'Chadic Lexical Roots'3) were devoted to Chadic reconstruction in the light of comparison with Afro-Asiatic.

The aim of this paper is to present new etymologies in addition to those Omotic lexemes whose etymologies have already been demonstrated by other authors. In the first part ${ }^{4}$ of this series, Omotic roots with * $b$ - plus dentals, sibilants, and velars were dealt with from an etymological standpoint. The second part (forthcoming) contains new etymologies for Omotic roots with * $b$ - followed by sonorants, etc. In this part I examine the Omotic lexical stock with the voiceless initial labial, of which (judging by evidence from Kefoid) there seems to have existed two varieties: ${ }^{*} p$ - vs. ${ }^{*} p^{h}$ - (reflecting the opposition of AA ${ }^{*} p$ - vs. ${ }^{*} f-$, resp.?). The numeration of the lexical entries is continuous, beginning from the very first paper.

\section{Omotic *p- and * $\mathrm{p}^{\mathrm{h}}$ -}

- 54. SOm.: Hamer pe 'earth, soil, ground' [Bnd. 1994, 148] II। (?) Eg. p '1. Untersatz, Sockel (aus Holz, Stein) (XVIII.), 2. Thron (GR)' (Wb I 489, 5-7) = '1. base (for statue) (XVIII.), 2. seat (Ptol.)' (EG 1927, 488, Q3) II। WCh.: Sura pèc '1. Grund, 2. Ursache’ [Jng. 1963, 78] I Pero péepè (redupl.) 'earth' [Frj. 1985, 45] II CCh.: Gude apaa (adv.) 'on the ground' [Hsk. 1983, 158] $<$ AA * $\sqrt{ } p$ 'ground' [GT].

- 55. NOm.: Kefoid ${ }^{*} \sqrt{ } \mathrm{p}^{\mathrm{h}} \mathbf{t}[\mathrm{GT}]:$ Kafa hot- [h- $<{ }^{*} \mathrm{p}^{\mathrm{h}}-<\mathrm{AA}{ }^{*} \mathrm{f}-$ ?] 'disprezzare, avvilire' [Crl. 1951, 457] = hot-èhè 'dispresso' [Cecchi apud Rn. 1888, 298] I I I Eg. ft 'sich ekeln, überdrüssig sein' (Med., Wb I 580, 8-13) = 'to show dislike, disgust' (FD 99) । I। PCh. *Vpt 'to refuse' [GT], a var. root displaying AA * $\sqrt{p t}[\mathrm{GT}]$, cf. CCh.: Bura mpimpata 'to scold, rebuke very severely' [BED 1953, 141] I Mofu-Gudur -pat- 'refuser catégoriquement de donner qqch. à qqn.' [Brt.

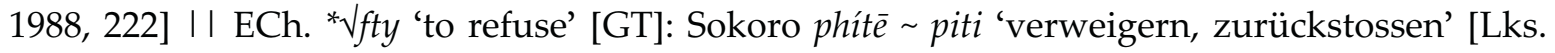
1937, 38] | WDangla pèètyè 'refuser de faire une chose' [Fédry 1971, 60], EDangla póótyé 'refuser, ne pas vouloir, résister, ne pas céder, ne pas admettre, ne pas accepter, récalcitrer' [Dbr.Mnt. 1973, 248] = póótyé 'ablehnen' [Ebs. 1979, 134; 1987, 73] | Birgit fòočí [-či < *-ti] 'refuser' [Jng. $1973 \mathrm{MS}$ ] $<\mathrm{AA} * \sqrt{f t} \sim * \sqrt{ } p t$ 'to despise' [GT].

- 56. Om. * put- 'cotton' [Bnd.] = *fūtt- 'cotton' [Lmb.] (Om.-Cu.: Crl. 1929, 29; 1938 III, 167, 202; 1951, 457; Bnd. 1988, 146; LS 1997, 357) III HECu. *fütt-a 'cotton' [Hds. 1989, 410; Sasse 1982, 71] | SOromo fút-a 'cotton' [Mrn.] | | | Eg. ftt (rope det.) 'ein pflanzlicher Faserstoff: als

${ }^{2}$ G. TAKÁCs. Outline of a North Bauchi Historical Phonology = Afrikanistische Arbeitspapiere 70 (2002), 167190. The Afro-Asiatic Background of the North Bauchi Consonant System II = TourneuX, H. (éd.). Topics in Chadic Linguistics III. Historical Studies. Papers from the $3^{\text {rd }}$ Biennial International Colloquium on the Chadic Languages, Villejuif, November 24-25, 2005. Köln, 2007., Rüdiger Köppe Verlag. Pp. 103-131.

${ }^{3}$ G. TAKÁCS. The 'Chadic Lexical Roots' and Their Afro-Asiatic Background Fourteen Years Later = BALDI, S. (ed.). Studi Magrebini. Vol. VII. Napoli, 2009. Pp. 211-224 (this part jointly with H. Jungraithmayr). The 'Chadic Lexical Roots' and Their Afro-Asiatic Background II = AwAGANA, A. \& LÖHR, D. (eds.). Topics in Chadic Linguistics VI: Papers from the $5^{\text {th }}$ Biennial International Colloquium on the Chadic Languages, Leipzig, June 11-13, 2009. Köln, 2011. Pp. 169-185. The 'Chadic Lexical Roots' and Their Afro-Asiatic Background III = BALDI, S. \& YAKASAI, H. M. (ed.). Proceedings of the $3^{\text {rd }}$ International Conference on Hausa Studies: African and European Perspectives (Napoli, $5^{\text {th }} 7^{\text {th }}$ July 2010). Studi Africanistici, Serie Ciado-Sudanese 3. Napoli, 2011. Pp. 313-329.

${ }^{4}$ G. TAKÁCS. Omotic Lexicon in its Afro-Asiatic Setting I: Omotic *b- with Dentals, Sibilants, and Velars = Busetto, L. (ed., scientific committee: M. Tosco, L. Tonelli, R. Sottile). He bitaney laagaa. Dedicato a/Dedicated to Marcello Lamberti. Quaderni di Lingua e Storia 3. Milano, 2011., Qu.A.S.A.R. s.r.1. Pp. 57-74.

${ }^{5}$ The origin of the Cu.-Om. terms for 'cotton' is disputed. E. Cerulli (1938 III, 167; 1951, 457) explained the HECu.-NOm. root from ES * $\sqrt{ } q^{w} t n$ 'cotton' < Ar. quṭ- 'cotton', which is phonologically unacceptable. M. M. Mo- 
Tampon-Zäpfchen, in Verbänden mit mrḥ.t und bj.t etc.' (Med., WÄDN 210-211, cf. also Wb I $581,9-10)=$ 'lint' (FD 99) = '1. Faserbausch (als Tampon-Zäpfchen), 2. Faserstoff (aus der Pflanze dbj.t): jrj $m$ ftt 'zum Faserbausch machen etwas (und in Körperöffnung einführen)' (GHWb 308) III NBrb.: Mzab ta-fatfat ta-fadfad-t, pl. ti-fatfat-in ti-fadfad-in '1. bourre de palmier, 2. plaque de cette bourre' [Dlh. 1984, 55-56] I I I CCh.: Muskum fít 'coton' [Trn. 1977, $20]^{6}<$ AA * $\sqrt[f t]{ }$ 'wadding, flock of plant (e.g. cotton)' [GT].

- 57. NOm.: PKefoid (Gonga) * ${ }^{\text {h }}$ ut- 'to smear' [GT]: Kafa hut- [h- < $p^{h}$-] 'ungere', hut-ō 'unguento' [Crl. 1951, 457] = hut- 'to smear, grease', hut-o 'ointment' [Flm.], Mocha pìtti-yé 'to anoint' [Lsl. 1959, 45], (?) Naga fur- [-r- <*-t-?] 'butter’ [d’Abbadie] (Gonga: Flm. 1987, 157, \#9) I I LEg. fdfd [< *ftft] 'vom Auftragen der Salbe an (r)' (GR hapax: Mendes stela, Urk. II 29, 12, Wb I 583, 4) = 'frotter avec de l'onguent' (Meeks, p.c. on 18 Feb. 2001) < AA * $\sqrt{f t}$ 'to anoint' [GT].

- 58. SOm.: Dime fot- 'to fall' [Bnd. 1994, 149] I I I ES: perhaps Gurage-Soddo fafat, Amh.

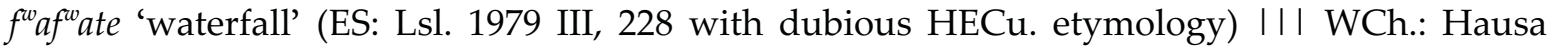
fáúčè [-če < *-te] '(bird) to swoop on to seize sg.' [Abr. 1962, 261] | | CCh.: Hurzo fat-day 'to descend' [Rsg.], Moloko fàtáy 'to descend' [Rsg.] (MM: Rsg. 1978, 236, \#189) I Lame putu 'tomber du haut de' [Scn. 1982, 272] II ECh.: Mubi fóót (pf.), Zafad-é (inf.), zufát (impf.) 'to fall' [Nwm. 1977, 183] = fádé (pl.) 'tomber' [Jng. 1990 MS, 14] (Ch.: JI 1994 II; Stl. 1996, 17) < AA * $[$ [p]t 'to fall' [GT]. ${ }^{7}$

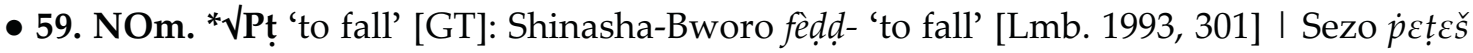
ṗغ̀țčšá 'he falls down' [Sbr.-Wdk. 1994, 12] III Sem.: Ar. fațara '(i.a.) jeter qqn. par la terre’ [BK II 609] I I | Ch. * Vpḍ 'to fall' [JS 1981, 98], in fact solely based on WCh.: Hausa fáádà 'to fall into, on', fáádád dà 'to drop sg., cause sg. to fall', fáádì 'to fall down, over, on to, into, off, out', fáádóó 'to fall down, off' [Abr. 1962, 241-243]< AA * $\sqrt{[} p]$ t 'to fall' [GT].

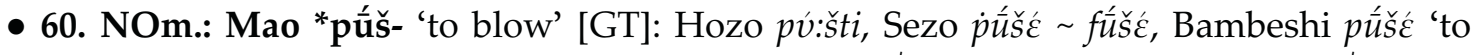
blow' (Mao gr.: Sbr.-Wdk. 1993, 13; 1994, 10) III Sem. * $\mid$ š 'to breathe, blow' vs. * $\sqrt{ }$ šw 'to break wind silently' [SED]: Akk. pašû G 'hauchen, leise furzen', Gtn 'immer wieder rasselnd atmen' [AHW 846] II PBHbr. Vpwš (met.) 'to breathe, rest one's self' [Jastrow 1950, 1149],

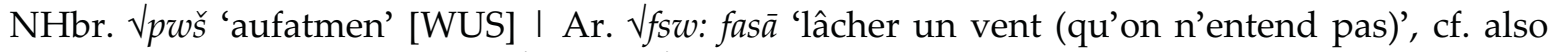
fiss-at- 'pet' [BK II 595] ${ }^{8} \mid$ I ES * $f_{f s w} \rightarrow{ }^{*} f^{w_{S}}$ ' to fart' [GT]: e.g. Geez fasawa 'to break wind' [Lsl.] vs. Tigre fäšfäšä 'to bluster, steam', fašfəšo 'vapour-bath' [Littmann-Höfner] (ES: Lsl. 1956, 199; 1987, 168; Sem.: SED I 314-5, \#56 vs. \#57) III CCh.: Kotoko fàssà 'souffle' [Bouny 1978, 57] | Mbara pìsé 'souffler' [TSL 1986, 275] I I ECh.: WDangla pî̀sè 'rester' [Fédry 1971, 64] < AA *V $p s$ '1. to blow, 2. breathe $\rightarrow$ rest, 3. fart' [GT]. ${ }^{10}$

- 61. NOm.: Yemsa (Janjero) fus- 'zwicken' [Lmb. 1993, 343] I I I WCh.: Angas-Sura *pus '1. to shoot (arrow), 2. sting' [GT]:11 e.g. Angas pus, pl. pwas '1. to shoot (as of an arrow),

reno $(1937,235)$ affiliated PCu.-Om. *fütt- 'cotton' with Sem.: Ar. füṭ-at- 'cotonata' [Mrn.] = 'serviette, essnie-mains' [Lsl.], which is improbable due to the supposed Indian origin of Ar. füț-at-, cf. OInd. pața- '(woven) cloth, blanket, garment, veil, etc.' [Monier-Williams 1899, 579] (as suggested by Vollers, ZDMG 50, 616 \& 56, 523, cf. also Leslau $1938,334 ; 1963,65)$.

${ }^{6}$ The Muskum parallel seems isolated in Chadic.

7 The uncertain reconstruction of PAA *[p]- is based on the * $p$ - in some remote root varieties.

${ }^{8}$ A var. root can be found in Ar. fašša 'faire sortir l'air d'une outre en la comprimant' [BK II 595].

9 The vocalism * $i$ - of the Chadic examples is obscure.

${ }^{10}$ Cf. also AA * $\sqrt{b s}$ '1. to blow, 2. breathe, 3. rest' [GT] (discussed in my paper 'Lexica Afroasiatica II', \#273).

${ }^{11}$ J. H. Greenberg $(1958,301)$, V. M. Illič-Svityč (1966, 25, \#3.9), followed by N. Skinner (1996, 66), correlated Angas-Sura *pus directly with OEg. pzh 'to bite (PT), sting (Med.)' (FD 94), a comparison rejected by G. Takács $(1999,82 ; 1999,368)$. The underlying PCh. root had a voiceless sibilant (cf. JI 1994 II, 2), although, in principle, the change Ch. ${ }^{*}$ Z $>$ Angas-Sura *-s seems plausible. 
2. shoot (of insects, of their stings), sting, 3. arrow' [Flk. 1915, 268-269] = puus '1. to shoot, 2. sting' [Grb.], Sura puus '1. to shoot, 2. sting' [Grb.] = pùs (sg.), pwas (pl.) 'schießen' [Jng. 1963, 79], Kofyar fús [fu- < *pu- reg.] 'to pierce' [Ntg. 1967, 13], Chip pùs '1. to shoot, 2. sting' [Grb.], Goemay puas (pl. of hes) 'to pierce' [Srl. 1937, 184] (AS: Grb. 1958, 301, \#2; Stl. 1977, 156, \#170) < AA * $\sqrt{p s}$ (possibly *pus-) '1. to sting, 2. shoot an arrow' [GT].

- 62. NOm.: Haruro pes-o pis-o ‘deretano’ [CR 1937, 657] I। I Eg. ps $\underline{d}$ [reg. < $\left.{ }^{*} p s g\right]^{12}$ 'Rücken, Rückgrat' (OK, Wb I 556, 1-9) = 'back, spine' (FD 95) I।। PCh. *pas- 'back' [GT]: WCh.: presumably Hausa fáásà 'to postpone (beginning sg.), be postponed indefinitely' [Abr. 1962, 257] II CCh.: Logone pásē ‘Gesäß, Hinterer' [Lks. 1936, 115] = mpásé 'cul' [Mch.] = páséé 'podex' [IS] < AA * $\sqrt{ }$ s (perhaps *pas- *pis-) 'back' [GT]..$^{13}$

- 63. NOm.: Zayse fīc-o [-ts-] 'tail' [Bnd.] = ficc-o [-ts-] 'tail' [Mkr.] = fiš-o 'tail' [Hyw. 1988, 285] = fic-o [-ts-] 'tail' [Sbr. 1994, 20] I I| Eth.-Sem. (borrowed from HECu.): Gurage: Selti fā̌čč, Zway afwaččo etc. 'tail, hair of tail' (ES: Lsl. 1979 III, 226) I I I HECu.: Burji fáčcoo 'bushy end of animal's tail' [Sasse 1982, 68: 'ohne Etymologie'], cf. Darasa fāč-ò fā̌ččo 'fly whisk' [Lsl.] । I CCh.: Logone pishaa 'tail' [Mkr.] < AA *pis- '2. tail' [GT]? Is it related to AA *Vps 'back' [GT] (above)?

- 64. SOm.: Ari feg-á 'far' [Bnd. 1994, 149] I I I ECu. *fVg- 'far' [Flm. 1969, 22, \#11]: LECu.: PSomali *fog 'far' [Ehret \& Nuuh Ali 1984]: Somali fog fug '1. Entfernung, 2. weit, fern' [Rn. 1902, 147] = fóg 'far' [Abr. 1964, 80], Rendille fog-á 'far' [Heine 1976, 215], Arbore feká fek-í [irreg. $-k<{ }^{*}-g$ ?] 'far' [Hyw. 1984, 358], Dasenech (Geleba) fik 'far, distant' [Flm./Dlg.] I Oromo fag-ō 'far' [Gragg 1982, 139] = fag-o? 'weit' [Sasse] I HECu.: Sidamo fag-o 'far' [Hds. 1989, 362] = faf- $\bar{o}\left[{ }^{*}{ }^{*} f a g^{w}{ }^{w}\right]$ 'lontano' [Crl. 1938 II, 200] (ECu.: CR 1913, 421; Dlg. 1973, 320; Sasse 1975, 246) I | | EBrb.: Ghadames $\underline{\underline{u}}-f \partial \dot{g}$ ' 1 . dépasser une limite, une mesure, 2. aller trop loin (ene verrou dans son logement)' [Lanfry 1973, 88, \#382] III WCh.: Ngizim fák [irreg. $-k<{ }^{*}-g$ ?] 'at a great distance (in time or space)' [Schuh 1981, 57] < AA * $\sqrt{P g}$ 'far, long' [GT].

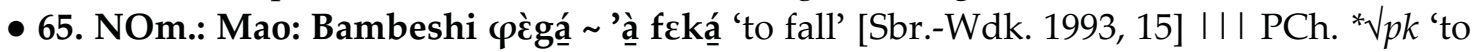
fall' [GT]: WCh.: Bade vg- 'fallen' [Lks. 1974-75, 105], Ngizim vàgú 'to fall down, descend down into, set (of sun)' [Schuh 1981, 165] = vàga 'to fall from high position' [JI] || CCh.: Bachama vùkó, fùkó 'to fall' [Skn.] = vúkó 'to fall' [Crn. 1975, 463] II ECh.: Jegu pak- 'herabfallen (Regen)' [Jng. 1961, 116] (Ch.: JI 1994 II, 130-131) < AA * $\sqrt{[} p] K$ 'to fall’ [GT].

- 66. NOm. * $\sqrt{\mathbf{p}^{\mathrm{h}} \mathbf{k}}$ 'to throw' [GT]: Janjero (Yemsa) foq- 'scagliare (la lancia)' [Crl. 1938 III, 73] = fòkà 'to throw spear' [Aklilu n.d.] | Mocha pòkkí-yé 'to throw away' [Lsl. 1959, 45] = p.ok'to throw away' [Flm.], Wombera hok- $a$ [* ${ }^{\mathrm{h}}{ }^{\mathrm{h}}$ ] 'rubbish, garbage' [Flm.] (Kefoid: Flm. 1987, 157) I । CCh. * $\sqrt{P k}$ 'to throw' [GT]: Gisiga $m u$-fko (nom. instr. prefix ma-) 'Wurfmesser' [Lks. 1970, 131] I Lame pík 'jeter (petites choses)' [Scn. 1982, 268], Zime-Dari pīk 'jeter (petites choses)' [Cooper 1984, 21] II ECh.: Tumak pòg 'abattre, faire tomber, terrasser (une personne...)' [Cpr. $1975,91]<\mathrm{AA} * \sqrt{ } P K$ 'to throw' [GT].

- 67. NOm.: Mao *Jp? 'to stab' [GT]: Hozo $p \bar{a} \sim p \bar{a}$ ' 'to stab, pierce', Sezo pe, pēe 'to stab, pierce', EMao piy-a 'to stab, pierce, kill' (Mao: Flm. 1988, 38) I I | WCh.: Ngizim vău '1. to shoot, 2. sting (scorpion)' [Schuh 1981, 167] I। CCh.: Mofu-Gudur váv vév (redupl.?) 'piquer (insecte)' [Brt. 1988, 251] < AA * $\sqrt{ } P H$ 'to sting' [GT].

- 68. SOm.: Hamer pi-o 'feces, dung' [Bnd. 1994, 149] II। SCu. * $p U[\varepsilon]$ - 'to defecate' [GT] = * $p \bar{u}\left\{-I^{*} p \bar{u} \mathcal{E}\right.$ - 'excrement' [Ehret]: Qwadza poro-tiko 'mud' I Ma'a ki-pwúlu 'excrement', -pwú?u 'to

12 The Egyptian counterpart has been extended by a third non-etymological * ${ }^{*}$ (hence - $\underline{d}$ ), which further occurs in a number of Eg. body part names, cf. e.g. fnd 'nose', mnd.t 'cheek', nhid.t 'tooth', hnd 'lower part, calf of leg' (meanings are quoted after FD). For the problem in detail cf. EDE II 577.

${ }^{13}$ Lit. for the AA comparison: Mkr. 1981, 115, \#24 (Gurage-Zayse); 1987, 360 (Logone-Burji-Zayse); Leslau 1979 III, 226; 1988, 188 (HECu.-Gurage). 
defecate' (SCu.: Ehret 1980, 145) I I CCh.: Nzangi poyai 'faeces' [Mch. in JI 1994 II, 129] < AA ${ }^{*} \sqrt{ } p \mathcal{\text { (or }}{ }^{*} \sqrt{ } \mathrm{q}$ ) ' 'to defecate' [GT]. ${ }^{14}$

- 69. NOm.: Janjero (Yemsa) fin- 'überqueren' [Lmb. 1993, 341] I I I LECu.: cf. perhaps Somali fān 'Prahlerei, Großtuerei, Lobgesang auf sich selbst' $[R n .1902,150]=$ fan-ayya 'to boast' [Abr. 1964, 76] I I I CCh.: Logone fánà fena 'übertreffen' [Lks. 1939, 91] = fan 'surpasser' [Mch.] I Matakam fana 'übertreffen' [Lks.], Mofu fun 'surpasser' [Mch. 1953, 187], Gisaga fun 'übertreffen' [Lks.] (Mafa-Mada: Lks. 1970, 35) < AA * $\sqrt{f n}_{n}$ (or ${ }^{*} \sqrt{p n}$ ?) 'to surpass' [GT]. ${ }^{15}$

- 70. NOm. *VVp(n)z 'ashes' [GT]: (?) Sheko fEmfus 'burnt' [Flm. 1972 MS, 1] I PMao *pūz[from *punz-?] 'ashes' [GT]: Bambeshi pùzè [Sbr.-Wdk. 1993, 13], Diddesa pūse [Flm. 1990, 27] = EMao pūse puse [Flm. 1988], Sezo pūsi jūsi [Flm. 1988] (Mao group: Flm. 1988, 38) II। LECu.: PSomali *be(ze)mbez [irreg. *b-] 'ashes' [Ehret \& Nuuh Ali 1984, 218] II। CCh. *pinž'ashes' [GT]: Tera pəžit [Nwm. 1964, 40, \#162], Pidlimdi pìż̀di [Krf.], Ga'anda fiža [Krf.] I Bura pinร̌u [BED 1953, 172] = pınร̌u [Krf.], Ngwahyi pınร̌u [Krf.], Margi pınsuḍu [Krf.] = ?pร̌ínẑduu? [JI], Chibak pınzù [Krf.] = pinzu [Ibr.] = panzu [IL] (CCh.: Krf. 1981, \#128; Ibr. 1990, 88; JI 1994 II, 4) < AA * $\sqrt{p n Z}$ 'ashes' [GT].

- 71. NOm.: Mao *pEng- 'to kill' [GT]: Hozo pèyg- 'to kill' [Flm.] = pengi [Bnd. 1994, 1158, \#43], Sezo piyaך 'to kill' [Flm.] (Mao: Flm. 1988, 38, \#1) I I I Eg. *png 'to kill (?)' (GT) $\rightarrow$ Cpt.: (B) ФWNX 'to overthrow, destroy' (CD 515a; CED 525) = 'vernichten, zerstören' (KHW 149) । I। CCh.: Mafa páng- 'égorger (plusieurs personnes, avec couteau)’ [Brt.-Bléis 1990, 308] < AA * $\sqrt{p n g}$ 'to kill' [GT].

- 72. NOm. *Jpng ‘to swim' [GT]: Oyda ìn̄ng- ‘to swim' [Bnd. 1971, 206] I Mao *pāyg- 'to swim' [GT]: Bambeshi pāyg- [Flm.-Bnd.] = pāyga [Mkr.], Diddesa pāyg- [Flm.-Bnd.], Hozo pāyg-

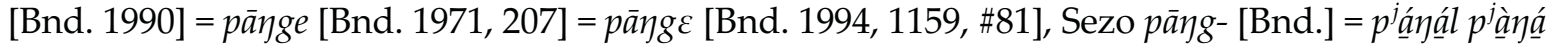

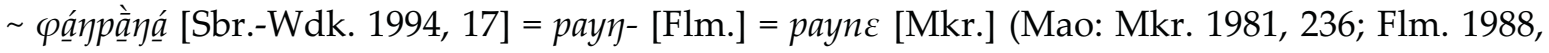
38; Bnd. 1990, 603, \#81) II। WCh.: SBauchi *pān(k)- 'to swim' [GT]: Boghom pàanki, Jum pàanak, Mangas paan, Kir pane (SBch.: Csp. 1994, 71) I I CCh.: Vulum fíní (adv. féng) [f < *b poss., vowel irreg.?] 'flotter' [Trn. 1978, 293], Pus fini 'flotter' [Trn. 1991, 88] ${ }^{16}<\mathrm{AA} * \sqrt{ } p n K$ 'to swim' [GT].17

- 73. NOm.: Mocha piripiro 'worm' [Lsl. 1959, 45]18, cf. probably Kafa hipper-ō [GT: < ${ }^{*} p^{h}$ irper- < * $p^{h}$ irp ${ }^{h}$ er- via dissim.?] 'verme' [Crl. 1951, 456] III Eth.-Sem. (borrowed from NOm.?): Gurage: Chaha, Ezha, Endegeny, Gogot, Selti farfar, Muher faraffar '1. kind of worm, 2. eggs of the tick, disease that affects the food' (Gurage: Lsl. 1979 III, 241: from NOm.?) III Eg. pizwj.w [<* prwj.w] (worm det.) (pl.) 'Bez. von Tieren die im Holz leben: Würmer (?), Ameisen (?)’ (XVIII, Wb I 498, 5) = ‘Art Tiere (die im/am Holz leben): Würmer oder Ameisen’ (GHWb 271) III NBrb.: Mzab ti-ffar-t ti-fra-t 'mites (insects)' [Dlh. 1984, 50] II| WCh.: Hausa fùùráú (m) 'larvae of digger-wasp' [Abr. 1962, 273] I। ECh.: EDangla pírpir̃è (m) 'le parasite du mil sorgho, 'coréides"' [Dbr.-Mnt. 1973, 245], Bidiya pirpị̀ [-ḍ < *-r-T?] 'ver de terre' [AJ 1989, 108] $<$ AA * $\sqrt{p r}$, possibly *pirpir- 'kind of worm' [GT].

- 74. NOm. *V $\mathbf{p}^{\mathrm{h}} \mathbf{r}$ 'hole' [GT]: Kafa hir-o [h- reg. < ${ }^{*} \mathrm{p}^{\mathrm{h}}-$ ] 'Loch' [Lmb.], Shinasha-Bworo furà 'Loch (zum Sehen)' [Lmb.] (NOm.: Lmb. 1993, 303) II। SAgaw: Awngi fár [irreg. f- < *p-] 'hole' [Apl. 1994 MS, 12.] II SCu.: Qwadza paral-uko [-1- reg. <*-r-] 'hole, pit' [Ehret 1980

${ }^{14}$ Represents a var. of AA * $\sqrt{ } \varepsilon \varsigma$ 'faeces, dirt' [GT]. For the AA etymology see also HSED \#179 (CCh.-SCu.); Ehret 1995, 91, \#39 (SCu.-Ngizim).

${ }^{15}$ A var. of AA $* \sqrt{b w n} \sim$ * $\sqrt{b n}$ 'to surpass' [GT] (see my paper 'Lexica Afroasiatica I' in AAP 2002, \#259).

${ }^{16}$ Weakening of PCh. * $-n k>{ }^{*} \eta$ in the Musgu group to *-ny?

${ }^{17}$ Cf. AA * $\sqrt{b} n \sim * \sqrt{ } b m$ 'to swim' [GT] (cf. my paper 'Lexica Afroasiatica I' in AAP 2002, \#131).

${ }^{18}$ C. T. Hodge $(1961,36)$ mistakenly identified the Mocha term with LEg. prpr 'to jump about' (q.v.) and Hausa pílpílò 'butterfly'. 
MS, 2] ${ }^{19}$ | || Sem.: Ar. fazara 'creuser la terre' [BK II 529] = 'to dig' [Lsl. 1987, 157] ${ }^{20}$ || ES: Gurage furä 'hole in the wall or in the fence of the house, hole in a container', cf. Ennemor \& Gyeto faräfärä 'to make a hole', Amh. färäffärä 'to make a hole', Tna. färfärä 'to pierce, break'

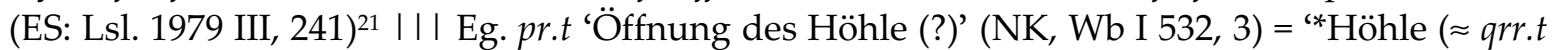
in anderen Versionen)' (GHWb 287) I I I NBrb.: Iznasen, Rif, Senhazha i-fri, pl. Iznasen, Tuzin, Uriaghel, Iboqqoyen i-fr-än, Ait Ammart i-farya-un, Senhazha i-frï-aw-en 'caverne, terrier, trou' (NBrb.: Rns. 1932, 298) III WCh.: Kofyar pigar piayar [<*pi $\gamma$ ar, $\left.-\gamma^{-}<{ }^{*}-2-\right]$ 'to bore a hole' [Ntg. 1967, 32] I Ngizim pàarú ‘to make holes with planting hoe to drop seeds in' [Schuh 1981, 132] II CCh.: Gude fóréey 'having one or more holes in it' [Hsk. 1983, 184] < AA * $\sqrt{f(2)} r$ * $\sqrt{ }(2) r^{22}$ 'to make a hole' [GT]. ${ }^{23}$

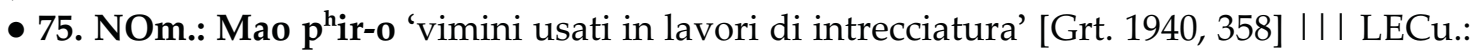
Baiso ferfer-a (f) 'punting pole, oar' [Hyw. 1979, 127-128] III Sem.: Hbr. *poßrāa(h), pl. por?ōt 'Ast, Zweig', půrā(h) 'Äste, Zweige' [GB 632] III SBrb.: ETawllemmet ā-fèr 'jonc' [Ncl. 1957, 56] III WCh.: Hausa firyáá fùryáá (f) 'small-headed drumstick' [Abr. 1962, 269, 274] < AA ${ }^{*} \operatorname{Pr}\left({ }^{*} \mathrm{p}\right.$ - or $\left.{ }^{*} \mathrm{f}-\right)$ 'stick, rod' [GT].

- 76. NOm.: Gimira pirik-o (?) 'ritorna! (imprv.)' [CR 1925, 622] II। Eth.-Sem.: Harari färäqa 'to turn', Amh. färäqa 'to turn' (ES: Lsl. 1963, 64) ||| Eg. pḥr [met. < *prḥ?] 'umwenden, umdrehen' (PT, Wb I 544-7) | I I NBrb.: Mzab a-fray '1. tourner, bifurquer, 2. être tordu, 3. (fig.) être mal venu, mal fait, défectueux' [Dlh. 1984, 52], Iznasen, Uriaghel, Senhazha e-fray 'être courbe, tordu, sinueux' [Rns. 1932, 299] II EBrb.: Ghadames e-fray 'être tordu' [Lanfry 1973, 98, \#430] II SBrb.: Ahaggar e-frey 'n'être pas droit (dévier de la ligne droite)' [Fcd. 1951-2, 3556] III CCh.: Mofu-Gudur -várk- '(se) retourner, tourner' [Brt. 1988, 250], Mafa vark- 'retourner (un récipient)' [Brt.-Bléis 1990, 370] I Vulum f 'r 'k ': firkì 'to turn upside down (renverser, re-

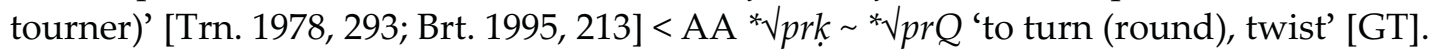

- 77. NOm.: NMao pēll-i 'vergine' [Grt. 1940, 358] I I Sem.: perhaps Akk. papallu (j/spB) 'Schößling, Zweig' [AHW 823] (unless it was a Sum. loan) (?) II। Dem. ppj [reg. < *ppl]

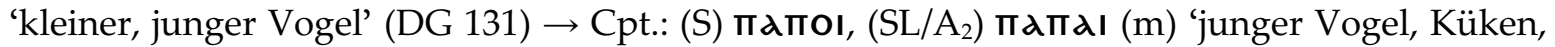
Huhn' (KHW 149) III WCh.: Angas-Sura *jal 'fresh, unripe' [GT]: Angas pal '1. unripe, 2. fresh sprouts or buds' [Flk. 1915, 259], Kofyar pél 'new, first' [Ntg. 1967, 32], Goemay pal 'unripeness' [Srl. 1937, 172] | SBauchi * $\bigvee_{p y l}$ 'new' [GT]: Guruntum pyàli [Csp.] = pyàalì [Jgr. 1989, 186], Tala pyaalii [Csp.] = pyaali [Smz.], Kir pyele [Csp.] = pyelè [Smz.], Laar pyella [Smz.], Mangas pyelà [Smz.] = pelàsà [Csp.], Soor (Zangwal) pyaalì [Smz.], Booluu pyaali [Smz.], Geji pyalì [Smz.], Zaranda pyààlè [Smz.], Zul pyel [Smz.], Barang \& Dir pyèlì [Smz.], Buli pyel [Smz.], Zeem pyàlì [Smz.] (SBch.: Smz. 1978, 44, \#96; Csp. 1994, 27, 60) II CCh.: Gude púl 'very new' [Hsk. 1983, 260] < AA * $\sqrt{ }$ l 'fresh, new' [GT].

${ }^{19}$ Ch. Ehret $(1980,143, \# 7)$ derived Qwadza paral-uko from his SCu. *pa\&- 'to cut', which is certainly false. By the way, Qwadza pazal- could be alternatively derived either from *pakar-, *pahar-, *pahar-or *paSar-.

${ }^{20}$ According to W. Leslau $(1987,157)$, the meaning of Ar. fazara is secondary, being a denominative verbal root derived from Ar. farr- 'rat, mouse', which would mean *'to dig as a rat does'. If so, its relatedness is improbable.

${ }^{21}$ W. Leslau (1979 III, 241) explained ES * $\sqrt{ }$ rffr 'to make a hole' (or sim.) [GT] from Sem. * $\sqrt{h} p r$ (with met. in Ar.-ES * $V_{f h r}$ ) 'to dig', which is disproved by the clear distinction in Tigrinya between the reflexes with and without *-h-, respectively, cf. Tna. färfärä 'to pierce' vs. fäharä 'to dig out'.

${ }^{22}$ Awngi $f$ - and Kefoid ${ }^{*} p^{h}$ - seem to point to AA ${ }^{*} f$ - $\neq$ AA ${ }^{*} p$ - indicated by Eg. $p$ - $=$ Qwadza $p$-. Perhaps there were two distinct var. roots (AA * $\sqrt{ } p r$ vs. * $f$ r $)$. Ch. Ehret $(1987, \# 189)$ combined Awngi far with ECu. *fur- 'to open'. Ultimately, a connection (at PAA level) to AA * $\sqrt{ } p r$ 'to open' (or sim.) cannot be excluded.

${ }^{23}$ Ch. Ehret $(1995,99, \# 61)$ equated the Ar.-Ngizim parallel with his PCu. "parr-/*barr- 'field, cultivated ground' and derived all of this from AA *-pâkr- 'to dig up', which is false. 
- 78. NOm.: Haruro (Kachama) pēl-uc [-ts] 'to pour' [Sbr. 1994, 18] | | | SCu.: Dahalo pill$\bar{e} \delta-$ 'to shake water off the body (in manner of dog or duck)' [Ehret 1980, 144; EEN 1989, 7] II I Sem.: Ug. pl 'rieseln' [WUS \#2219], Syr. ฟpll 'to sprinkle' [Lsl.] I I Ar. Vfyl 'vergießeln, rieseln' [WUS] II ES: Geez falfala 'to gush out, spring forth, bubble up, break forth, burst out as a fountain, make gush, etc.' [Lsl. 1987, 158] III ECh.: WDangla pâllè 'asperger (équivalent profane de bâlllè, réservé aux libations faites aux génies)' [Fédry 1971, 58] < AA * $p l$ 'to sprinkle' [GT].

- 79. NOm.: Benesho pel 'to blow (e.g. on fire)' [Wdk. 1990, 109] III LECu.: Afar fulto 'blowing, puffing' [PH 1985, 105] | Oromo fōl-ī 'odor', fōll-a $a$ a 'to have odor, give scent' [Gragg 1982, 148] = fōl-i 'odore' [Mrn.] | HECu. * fōl- 'to breathe', 'fōl-e 'breath, odor' [Hds.]: Sidamo fōl- fō $2 l$ - 'to breathe', fōl-e 'breath, odor, smell' [Hds.] = fōl- $\bar{a}$ 'odore' [Crl. 1938 II, 200] = fōl-é 'spirito' [Mrn. 1940, 215], Burji fōl-e 'odor, smell' [Hds.], Hadiya fōša [< ‘fōl-ča] 'odor, smell' [Hds.] (HECu.: Hds. 1989, 106-107, 363, 409; Lsl. 1956, 993) I। SCu.: perhaps Qwadza pelay-iko [p- irreg., -iko noun suffix] 'wind' [Ehret 1980, 144] I। I Eth.-Sem. (borrowed from ECu.?): Gurage: Wolane \& Masqan fol 'breath' [Lsl. 1979 III, 231] I I I WCh.: Daffo-Butura fòl 'Pfeife' [Jng. 1970, 214] I Jimbin fâl- 'to blow' [Skn.] I Kir fwale 'to blow (mouth)' [Csp. 1994, 42], Guruntum fali 'to blow' [Jgr. 1989, 183] II CCh.: Zime fòl 'to blow' [CWC in Mkr.] I Pus fili 'vanner au vent' [Trn. 1991, 88] I I ECh.: Kera fúulí 'blasen, wehen' [Ebert 1976, 46] = f́ććlé 'to blow' [Ebert in JI] (Ch.: Mkr. 1987, 103; JI 1994 II, 32-33) < AA * $V_{f l}$ '1. to blow, 2. smell, 3. breathe' [GT].

- 80. NOm.: Kafa pay-ō 'canna con la quale si danno battiture' [Crl. 1951, 481] I I I Eg. pjpj.t (wood det.) 'der Kielbalken des Schiffes (?)' (NE, Wb I 502, 8) = 'keel' (DLE I 170) = 'la quille (?) d'un navire' (AL 79.0973) = “*Kiel' (GHWb 273) I I I Bed. fu (f) 'die große Zeltstange in der Mitte des Zeltes' [Rn. 1895, 75] = fi (f), pl. fō-t '1. pole, prop, esp. of interior, 2. (pars pro toto) house, home' [Rpr. 1928, 177] I I I CCh.: Hina pai '1. Baum, 2. Mattenstange' [Str.], cf. Daba pă poй 'Baum' [Str.] (CCh.: Str. 1922-3, 129, 136) < AA * لpy '(log of) wood' [GT].

- 81. SOm.: Dime fuy- '1. to spit, 2. saliva' [Bnd. 1994, 159] I I SCu. *para- 'spit, sputum' [Ehr.]: Ma'a ma-pazé 'spit, sputum' [Ehr. 1980, 143] III Sem.: Ar. Vfwీ, impf. yafū $\{u$ 'speien, vomieren' [Vcl.] I I L LE. $p \varepsilon$ (or $p\{j$ ) 'spucken' (GR, Edfu II 260, 12, Grdseloff, ArOr 20, 1952, 482-486) = 'cracher' (Drioton, RdE 10, 1955, 91-92; AL 77.1381) = 'speien' (NB̈̈ 195) । I। WCh.: Tangale puye 'to expectorate, eject from mouth (anything unpleasant)' [Jng. 1991, 135] < AA * $\sqrt{ } \mathcal{L}$ (presumably *pu\&-) 'to spit' [GT]. Onomatopoeic. See also Vcl. 1959, 73; 1959, 39; 1959, 29 (Eg.-Ar.); Takács 1998, 158, \#4.3 (Eg.-Ar.-Ma’a); 2000, 75, \#2.6 (Ma’a-Ch.-Ar.).

\section{Special symbols}

$P$ : any labial stop $(f, p, b, \dot{p}), T$ : unspecified dental stop $(t, d, t), S$ : any voiceless sibilant and/or affricate $(s, \breve{s}, \hat{s}, c, \breve{c}, \hat{c})$, $Z$ : unspecified voiced sibilant and/or affricate $(z, 3, \breve{3}), K$ : any velar stop $(k, g, k), Q$ : unspecified uvular or postvelar etc. $(q, g, \dot{q}, h), \mathrm{H}$ : any of the pharyngeals or laryngeals etc. $(\{, \gamma, h, h, \imath)$. The vertical strokes signify the degree of closeness of the language groups (e.g. Kotoko I Masa), subbranches (e.g. North Berber II East Berber), and branches (Semitic I I Egyptian), from which the individual lexical data are quoted.

\section{Abbreviations of languages and other terms}

(A): Ahmimic, AA: Afro-Asiatic (Afrasian, Hamito-Semitic), Akk.: Akkadian, Alg.: Alagwa, Amh.: Amharic, Ar.: Arabic, Aram.: Aramaic, Ass.: Assyrian, (B) Bohairic, Bab.: Babylonian, BAram.: Biblical Aramaic, Bed.: Bed'awye (Beja), Brb.: Berber (Libyo-Guanche), Brg.: Burunge, C: Central, Ch.: Chadic, Cpt.: Coptic, CT: Coffin Texts, Cu.: Cushitic, Dem.: Demotic, Dhl.: Dahalo, E: East, Eg.: Egyptian, ES: Ethio-Semitic, ESA: Epigraphic South Arabian, Eth.: Ethiopian, Eth.-Sem.: Ethio-Semitic, (F): Fayyumic, GR: Ptolemaic and Roman period, Grw.: Gorowa, 
H: Highland (in Cushitic), Hbr.: Hebrew, Hgr.: Ahaggar, Hrs.: Harsusi (in MSA), Irq.: Iraqw, Jbl.: Jibbali, L: Late, L: Low(land), lit.: literature, LP: Late Period, M: Middle, Mag.: magical texts, Med.: medical texts, MK: Middle Kingdom, MSA: Modern South Arabian, N: New, N: North, NE (or NEg.): New Egyptian, NK: New Kingdom, O: Old, OK: Old Kingdom, Om.: Omotic, Omt.: Ometo, P: Proto-, PB: Post-Biblical, PT: Pyramid Texts, reg.: regular, S: South, (S): Sahidic, Sem.: Semitic, Sqt.: Soqotri, Syr.: Syriac, Ug.: Ugaritic, W: West, Wlm(d).: Tawllemmet.

\section{Abbreviations of author names}

Abr.: Abraham, AJ: Alio \& Jungraithmayr, Akl.: Aklilu, Alm.: Alemayehu, Apl.: Appleyard, BK: Bieberstein \& Kazimirski, Blz.: Blažek, Bnd.: Bender, Brn.: Brunet, Brt.: Barreteau, Cpr.: Caprile, CR: Conti Rossini, Crl.: Cerulli, Crn.: Carnochan, Csp.: Cosper, Dbr.: Djibrine, Dlg.: Dolgopolsky, Dlh.: Delheure, Ebs.: Ebobisse, EEN: Ehret \& Elderkin \& Nurse, Ehr.: Ehret, Fcd.: Foucauld, Fdr.: Fédry, Flk.: Foulkes, Flm.: Fleming, Frj.: Frajzyngier, Frz.: Fronzaroli, GB: Gesenius \& Buhl, Grb.: Greenberg, Grt.: Grottanelli, GT: Takács, Hds.: Hudson, Hsk.: Hoskison, Hyw.: Hayward, Ibr.: Ibriszimow, IL: Institute of Linguistics, IS: Illič-Svityč, JA: Jungraithmayr \& Adams, Jgr.: Jaggar, JI: Jungraithmayr \& Ibriszimow, Jng.: Jungraithmayr, JS: Jungraithmayr \& Shimizu, Krf.: Kraft, Lks.: Lukas, Lmb.: Lamberti, Lnf.: Lanfry, Lsl.: Leslau, Mch.: Mouchet, Mkr.: Mukarovsky, Mlt.: Militarev, Mnt.: Montgolfier, Mrn.: Moreno, Ncl.: Nicolas, Ntg.: Netting, Nwm.: Newman, PH: Parker \& Hayward, Rn.: Reinisch, Rns.: Renisio, Rpr.: Roper, Rsg.: Rossing, Rsl.: Rössler, Sbr.: Siebert, Scn.: Sachnine, Skn.: N. Skinner, Smz.: Shimizu, Srl.: Sirlinger, Ss.: Sasse, Stl.: Stolbova, Str.: Strümpell, Trn.: Tourneux, TSL: Tourneux \& Seignobos \& Lafarge, Vcl.: Vycichl, Wdk.: Wedekind, Zbr.: Zaborski, Zhl.: Zyhlarz.

\section{Literature}

AAP = Afrikanistische Arbeitspapiere (Köln).

AbraHAM, R. C.: Dictionary of the Hausa Language. ${ }^{2}$ London, 1962, University of London Press.

ABRAHAM, R. C.: Somali-English Dictionary. ${ }^{2}$ London, 1964, University of London Press Ltd.

AHW = SODEN, W. von: Akkadisches Handwörterbuch. I-III. Wiesbaden, 1965-1981, Otto Harrassowitz.

AKLILU, Y.: Yem wordlist. MS. Not dated.

AL I-III = MeEKS, D.: Année lexicographique. Égypte ancienne. Tome 1-3 (1977-1979). 2 ème édition. Paris, 1998, Cybele. Alio, Kh. \& JungraithmaYr, H.: Lexique bidiya. Frankfurt am Main, 1989, Vittorio Klostermann.

APPleyARD, D.: English-Awngi \& Awngi-English Wordlists. Drawn from HETZRON, 'The Verbal System of Southern Agaw', 1969, and Hetzron, 'The Nominal System of Awngi (Southern Agaw)', BSOAS, 1978, together with additional material collected by D.A. in 1987-1991. MS. London, 1994. 16 p.

ArOr = Archív Orientální (Praha).

BARreteau, D.: Description d mofu-gudur. Langue de la famille tchadique parlée au Cameroun. Livre II. Lexique. Paris, 1988, Éditions de l'ORSTOM.

BArreteau, D.: Vowel and Tonal Variations within the Consonantal Framework of the Verbal System in Central Chadic Languages. In: IBriszimow, D. \& LEGER, R. (eds.): Studia Chadica et Hamitosemitica. Köln, 1995, Rüdiger Köppe Verlag. Pp. 197-228.

BED = ANONYMOUS: Bura-English Dictionary. (Place unknown), 1953, (publisher unnamed). Master copy in the library of the Seminar für Afrikanische Sprachen und Kulturen der Universität Hamburg (inv. no.: 15748 / JT 1526).

Bender, M. L.: The Languages of Ethiopia. A New Lexicostatistic Classification and Some Problems of Diffusion. In: Anthropological Linguistics 13/5 (1971), 165-288.

Bender, M. L.: Omotic: A New Afroasiatic Language Family. Carbondale, Illinois, 1975, Southern Illinois University.

Bender, M. L.: Proto-Omotic Phonology and Lexicon. In: BechHAus-Gerst, M.; Serzisko, F. (eds.): Cushitic-Omotic. Papers from the First International Symposium on Cushitic and Omotic Languages, Cologne, January 6-9, 1986. Hamburg, 1988, Helmut Buske Verlag. Pp. 121-159.

Bender, M. L.: The Limits of Omotic. In: HAYWARD, R. J. (ed.): Omotic Language Studies. London, 1990, SOAS. Pp. 584-616.

BENDER, M. L.: Aroid (South Omotic) Lexicon. In: Afrikanistische Arbeitspapiere 38 (1994), 133-162. 
Bender, M. L.: The Mystery Languages of Ethiopia. In: MARcus, H. (ed.): New Trends in Ethiopian Studies. Vol. 1. Lawrenceville, 1994, Red Sea Press. Pp. 1153-1174.

Bender, M. L.: The Omotic Languages: Comparative Morphology and Lexicon. München, 1999, Lincom Europa.

Bender, M. L.: Synthesis of the Northwest Ometo Dialect Cluster. Chart 1: Selected Lexical Items of the Welaitta Cluster. Preliminary extracts from the author's preparing Omotic Comparative Lexicon. MS. Carbondale, Illinois, 1999. Pp. 8-37.

BENDER, M. L.: Omotic Lexicon and Phonology. Carbondale, 2003, SIU Printing / Duplicating, Southern Illinois University.

Biberstein KAZIMIRSKI, A. de: Dictionnaire arabe-français. I-II. Paris, 1860, Maisonneuve et Cie.

BLAŽEK, V.: Omotic Lexicon in Afroasiatic Perspective: Body Parts Cognates. MS. Paper presented at the $2^{\text {nd }}$ International Symposium on Cushitic and Omotic Languages (Torino, November 1989). 41 p.

Blažek, V.: Bedawye Etymologies. MS. Př́ibram, around 1990. 10 p.

BLAŽEK, V.: Toward the Position of Bed'awye within Afroasiatic. An Analysis of the Body Parts Terminology. MS. Printout in Köln, March 1994. 49 p.

BLAŽEK, V.: Fragment of a Comparative and Etymological Dictionary of Beja Anatomical Lexicon. MS. 2000. 90 p.

BLAŽEK, V.: Beja Colour Terminology in a Comparative-Etymological View. MS. 2000. 5 p.

BLAŽEK, V.: Beja Kinship and Social Terminology. MS. 2000. 11 p.

BLAŽEK, V.: Flora in Beja Lexicon. MS. 2000. 8 p.

BLAŽEK, V.: Fauna in Beja Lexicon. MS. 2000. 27 p.

BLAŽEK, V.: Fauna in Beja Lexicon. A Fragment of a Comparative-Etymological Dictionary of Beja. In: KogAN, L. (ed.): Orientalia: Papers of the Oriental Institute. Issue III. Studia Semitica. Moscow, 2003, Russian State University of Humanities. Pp. 230-294.

BLAŽEK, V.: Natural Phenomena, Time and Geographical Terminology in Beja Lexicon. Fragment of a Comparative and Etymological Dictionary of Beja: I. In: Babel und Bibel 2 (2006), 365-407.

BOUnY, P.: La formation du pluriel des nominaux en kotoko. In: CAPRILE, J.-P. \& JUNGRAITHMAYR, H. (eds.): Préalables à la reconstruction du proto-tchadique. Paris, 1978, SELAF. Pp. 51-65.

CAPRILE, J.-P.: Lexique tumak-français (Tchad). Berlin, 1975, Verlag von Dietrich Reimer.

CARnochan, J.: Bachama and Chadic. In: BYnON, J.; BYnON, Th. (eds.): Hamito-Semitica. The Hague, 1975, Mouton. Pp. 459-468.

CD = CRuM, W. E.: A Coptic Dictionary. Oxford, 1939, Oxford, 1939, Oxford University Press.

CED = ČERNÝ, J.: Coptic Etymological Dictionary. London, Cambridge, 1976, Cambridge University Press.

CERULli, E.: Note su alcune popolazioni sidāmā dell'Abissinia meridionale II: i Sidama dell'Omo. In: Rivista degli Studi Orientali 12 (1929), 1-69.

Cerulli, E.: Studi etiopici. II. La lingua e la storia dei Sidamo. Roma, 1938, Istituto per l'Oriente.

CERUlLI, E.: Studi etiopici. III. Il linguaggio dei Giangerò ed alcune lingue Sidama dell'Omo (Basketo, Ciara, Zaissè). Roma, 1938, Istituto per l'Oriente.

Cerulli, E.: Studi etiopici. IV. La lingua caffina. Roma, 1951, Istituto per l'Oriente.

CLD I-III = STOLbOVA, O.: Chadic Lexical Database. Moscow-Kaluga, 2005-9, Diaphragma.

CONTI RossinI, C.: Studi su populazioni dell'Etiopia. In: Rivista degli Studi Orientali 6 (1913), 365-426.

Conti Rossini, C.: Sui linguaggi dei Naa e dei Ghimirra (Sce) nell’Etiopia Meridionale. In: Rendiconti della Reale Accademia dei Lincei, Classe di Scienze morali, storiche e filologiche, ser. VI, vol. 1 (1925), 512-636.

COOPER, K. N.: Lexique zime-français. Vūn tàrí. Sarh (Tchad), 1984, Centre d'Études Linguistiques.

COSPER, R.: South Bauchi Lexicon. A Wordlist of Nine South Bauchi (Chadic) Languages and Dialects. Halifax, 1994, The Author (Saint Mary's University).

$\mathrm{CWC}=$ Chadic Word Catalogue (Marburg, Frankfurt a/M).

DelHeUre, J.: Dictionnaire mozabite-français. Paris, 1984, Société d'Études Linguistiques et Anthropologique de France (SELAF).

DG = ERICHSEN, W.: Demotisches Glossar. Koppenhagen, 1954, Ejnar Munksgaard.

Djibrine, B. A. Z. \& Montgolfier, P. de (etc.): Vocabulaire dangaléat. Kawo dayla. (Place not indicated), around 1973, (publisher not indicated).

DOLGOPOL'SKIJ, A. B.: Sravnitel'no-istoričeskaja fonetika kušitskih jazykov. Moskva, 1973, Nauka.

DOlgopolsKy, A.: Semitic and East Cushitic. Sound Correspondences and Cognate Sets. In: SEGERT, S. \& BODROGLIGETI, A. J. E. (eds.): Ethiopian Studies Dedicated to Wolf Leslau. Wiesbaden, 1983, Otto Harrassowitz. Pp. $123-142$. 
DOlgOPOLSKY, A.: South Cushitic Lateral Consonants as Compared to Semitic and East Cushitic. In: JUNGRAithmayr, H. \& Müller, W. W. (eds.): Proceedings of the Fourth International Hamito-Semitic Congress. Amsterdam, 1987, John Benjamins. Pp. 195-214.

DOlgOPOLSKY, A.: Semitic and East Cushitic: Word-Initial Laryngeals. In: TADDESE, B. (ed.): Proceedings of the Eighth International Conference of Ethiopian Studies, University of Addis Ababa, 1984. Volume 1. Addis Ababa, 1988, Institute of Ethiopian Studies, Addis Ababa. Pp. 629-637.

EBERT, K. H.: Sprache und Tradition der Kera (Tschad). Teil II. Berlin, 1976, Dietrich Reimer.

EboBISSE, C.: Die Morphologie des Verbs im Ost-Dangaleat (Guera, Tschad). Berlin, 1979, Dietrich Reimer Verlag.

EBOBISSE, C.: Les verbaux du dangaléat de l'est (Guera, Tchad). Lexiques français-dangaléat et allemand-dangaléat. Berlin, 1987, Dietrich Reimer Verlag.

EDE I = TAKÁCS, G.: Etymological Dictionary of Egyptian. Volume One: A Phonological Introduction. Leiden, 1999, E. J. Brill.

EDE II = TAKÁCS, G.: Etymological Dictionary of Egyptian. Volume Two: $b$-, p-, f-. Leiden, 2001, E. J. Brill.

Edfu (Edfou) II-XIV = CHASsINAT, E.: Le temple d'Edfou. Tome II-XIV. Le Caire, 1918, 1928-34, Leroux.

EG 1927 = GARDINER, A. H.: Egyptian Grammar. ${ }^{1}$ Oxford, 1927, Clarendon Press.

EHRET, Ch.: The Historical Reconstruction of Southern Cushitic Phonology and Vocabulary. Berlin, 1980, Dietrich Reimer Verlag.

EHret, Ch. \& Ali, M. N.: Soomaali Classification. In: LABAHN, T. (ed.): Proceedings of the Second International Congress of Somali Studies. Vol. 1. Hamburg, 1984, Buske Verlag. Pp. 201-269.

EHRET, Ch.: Proto-Cushitic Reconstruction. In: Sprache und Geschichte in Afrika 8 (1987).

Ehret, Ch. \& Elderkin, E. D. \& NurSe, D.: Dahalo Lexis and Its Sources. In: Afrikanistische Arbeitspapiere 18 (1989), 5-49.

FD = FAULKNER, R. O.: A Concise Dictionary of Middle Egyptian. Oxford, 1962, Clarendon Press.

FÉDRY, J. (avec la collaboration de KHAMIS, J. \& O/NEDJEI, M.): Dictionnaire dangaleat (Tchad). Thése de $3^{\text {3eme }}$ cycle, Institut National des Langues et Civilisations Orientales. Lyon, 1971, Afrique et Langage.

FLEMING, H. C.: Asa and Aramanik: Cushitic Hunters in Masai-Land. In: Ethnology 8/1 (1969), 1-36.

FLEMING, H. C.: Sheko Word List. MS. Ca. 1972. 10 p.

Fleming, H. C.: Proto-Gongan Consonant Phonemes: Stage One. In: MuKarovsky, H. G. (ed.): Leo Reinisch. Werk und Erbe. Wien, 1987, Verlag der Österreichischen Akademie der Wissenschaften. Pp. 141-159.

Fleming, H. C.: Mao's Ancestor. Consonant Phonemes of Proto-Mao. Stage One. In: GrOMYKO, A. A. (ed.): Proceedings of the Ninth International Congress of Ethiopian Studies (Moscow, 26-29 August 1986). Vol. 5. Moscow, 1988, Nauka. Pp. 35-44.

Fleming, H. C.: Proto-South-Omotic or Proto-Somotic Consonant Phonemes: Stage One. In: BechHAUS-GeRST, M. \& SERZISKO, F. (eds.): Cushitic-Omotic. Papers from the International Symposium on Cushitic and Omotic Languages, Cologne, January 6-9, 1986. Hamburg, 1988, Helmut Buske Verlag. Pp. 163-175.

Fleming, H. C.: Omotica, Afrasiana and More: Ethiopia as the Ever-Flowing Vase. In: Mother Tongue 12 (1990), $22-30$.

FoucAuld, Ch. de: Dictionnaire touareg-français, dialecte de l'Ahaggar. Vol. I-IV. Paris, 1951-1952, Imprimerie Nationale de France.

FoulKes, H. D.: Angass Manual. Grammar, Vocabulary. London, 1915, Kegan Paul, Trench, Trübner and Co.

FrAJZYNGIER, Z.: A Pero-English and English-Pero Vocabulary. Berlin, 1985, Dietrich Reimer Verlag.

GHWb = HANNIG, R.: Grosses Handwörterbuch Ägyptisch-Deutsch (2800-950 v. Chr.). Mainz, 1995, Verlag Philipp von Zabern.

GRAGG, G.: Oromo Dictionary. East Lansing, Michigan, 1982, Michigan State University.

GreenberG, J. H.: The Labial Consonants of Proto-Afro-Asiatic. In: Word 14 (1958), 295-302.

Grottanelli, V. L.: Missione etnografica nel Uollega Occidentale. Volume primo. I Mao. Roma, 1940, Reale Accademia d'Italia.

HAYWARD, R. J.: Bayso Revisited: Some Preliminary Linguistic Observations. II. In: Bulletin of the School of African and Oriental Research 62 (1979), 101-132.

HAYWARD, R. J.: The Arbore Language: A First Investigation Including a Vocabulary. Hamburg, 1984, Helmut Buske Verlag.

Hayward, R. J.: Remarks on Omotic Sibilants. In: Becchaus-Gerst, M. \& SERZISKO, F. (eds.): Cushitic-Omotic. Papers from the International Symposium on Cushitic and Omotic Languages, Cologne, January 6-9, 1986. Hamburg, 1988, Helmut Buske Verlag. Pp. 263-299. 
HeINE, B.: Notes on the Rendille Language. In: Afrika und Übersee 59 (1976), 176-223.

Hodge, C. T.: Review of Leslau, W.: A Dictionary of Mocha (Southwestern Ethiopia). In: African Studies 20 (1961), 113.

Hoskison, J. T.: A Grammar and Dictionary of the Gude Language (Chadic). Ph.D. dissertation. 1983, The Ohio State University.

HSED = Orel, V. É.; STOLbova, O. V.: Hamito-Semitic Etymological Dictionary. Leiden, 1995, E. J. Brill.

Hudson, G.: Highland East Cushitic Dictionary. Hamburg, 1989, Buske.

IBRISZIMOW, D.: Towards a Common Chadic Lexicon. In: Zeszyty Naukowe Universytetu Jagiellońskiego. Prace językoznawcze 102 (1990), 1-122.

IL = Institute of Linguistics. Bauch Area Survey Report presented by N. Campbell and J. Hoskison. MS. Zaria, 1972.

ILLIČ-SVITYČ, V. M.: Iz istorii čadskogo konsonantizma. Labial’nye smyčnye. In: UsPENSKIJ, B. A. (ed.): Jazyki Afriki. Voprosy struktury, istorii i tipologii. Moskva, 1966, Nauka. Pp. 9-34.

JagGAR, Ph. J.: Guruntum (gùrdùn) (West Chadic-B): Linguistic Notes and Wordlist. In: African Languages and Cultures 2/2 (1989), 175-202.

JASTROW, M.: A Dictionary of the Targumim, the Talmud Babli and Yerushalmi, and the Midrashic Literature. Volume I: 2-k, Volume II: $l-t$. New York, 1950, Pardes Publishing House Inc.

JUNGRAITHMAYR, H.: Beobachtungen zur tschadohamitischen Sprache der Jegu (und Jonkor) von Abu Telfan (Republique du Tchad). In: Afrika und Übersee 45 (1961), 95-123.

Jungraithmayr, H.: Die Sprache des Sura (Maghavul) in Nordnigerien. In: Afrika und Übersee 47 (1963), 8-89, 204220.

JungraithmayR, H.: Die Ron-Sprachen. Tschadohamitische Studien in Nordnigerien. Glückstadt, 1970, Verlag J. J. Augustin.

JUNGRAITHMAYR, H.: Dictionnaire birgit-français. MS. 1973.

JungratthmaYR, H. \& SHIMIZU, K.: Chadic Lexical Roots. Vol. II. Tentative Reconstruction, Grading and Distribution. Berlin, 1981, Verlag von Dietrich Reimer.

JUNGRAITHMAYR, H.: Lexique mokilko. Berlin, 1990, Dietrich Reimer Verlag.

JUNGRAITHMAYR, H.: Lexique mubi-français (Tchad oriental). MS. Frankfurt a/M, 1990. 50 p.

JUnGRAithMAYR, H. (in collaboration with N. A. GALADIMA and U. KLEINEWILlinghÖFER): A Dictionary of the Tangale Language (Kaltungo, Northern Nigeria) with a Grammatical Introduction. Berlin, 1991, Dietrich Reimer Verlag.

Jungraithmayr, H. \& Ibriszimow, D.: Chadic Lexical Roots. Volume II. Documentation. Berlin, 1994, Dietrich Reimer Verlag.

KHW = WESTENDORF, W.: Koptisches Handwörterbuch. Heidelberg, 1977, Carl Winter Universitätsverlag.

KRAFT, Ch. H.: Chadic Wordlists. I-III. Berlin, 1981, Dietrich Reimer Verlag.

LAMBERTI, M.: Die Shinassha-Sprache. Materialien zum Boro. Heidelberg, 1993, Carl Winter Universitätsverlag.

LAMBERTI, M.: Materialien zum Yemsa. Heidelberg, 1993, Carl Winter Universitätsverlag.

LAmberti, M. \& Sottile, R.: The Wolaytta Language. Köln, 1997, Rüdiger Köppe Verlag.

LANFry, J.: Ghadames II. Glossarie. Alger, 1973, Le Fichier Périodique.

LESLAU, W.: Lexique soqoțri (sudarabique moderne), avec comparaisons et explications étymologiques. Paris, 1938, Librairie C. Klincksieck.

LeSLAU, W.: Gafat Documents. Records of a South-Ethiopic Language. Grammar, Text and Comparative Vocabulary. New Haven, Connecticut, 1945, American Oriental Society.

LESLAU, W.: Étude descriptive et comparative du gafat (éthiopien méridional). Paris, 1956, Librairie C. Klincksieck.

LeSLAU, W.: Additional Notes on Kambatta of Southern Ethiopia. In: Anthropos 51 (1956), 985-993.

Leslau, W.: A Dictionary of Moča (Southwestern Ethiopia). Berkeley, Los Angeles, 1959, University of California Press.

LESLAU, W.: Etymological Dictionary of Harari. Berkeley, Los Angeles, 1963, University of California.

Leslau, W.: Southeast Semitic Cognates to the Akkadian Vocabulary. III. In: Journal of the American Oriental Society 89 (1969), 18-22.

LESLAU, W.: Etymological Dictionary of Gurage (Ethiopic). Vol. III. Etymological Section. Wiesbaden, 1979, Otto Harrassowitz.

LeSlau, W.: Comparative Dictionary of Gerez (Classical Ethiopic). Wiesbaden, 1987, Otto Harrassowitz.

LESLAU, W.: Observations on Sasse's Vocabulary of Burji. In: Afrika und Übersee 71 (1988), 177-203.

LUKAS, J.: Die Logone-Sprache im Zentralen Sudan. In: Abhandlungen für die Kunde des Morgenlandes 21/6 (1936). 
LUKAS, J.: Zentralsudanische Studien. In: Abhandlungen aus dem Gebiet der Auslandskunde. Hansische Universität. Reihe B, Band 45, Band 24 (1937).

LUKAS, J.: Die Sprache des Buduma in Zentralen Sudan. In: Abhandlungen für die Kunde des Morgenlandes 24/2 (1939).

LUKAS, J.: Studien zur Sprache der Gisiga (Nordkamerun). Hamburg, 1970, Verlag J. J. Augustin.

LUKAS, J.: Studien zur Bade-Sprache (Nigeria). In: Afrika und Übersee 58 /2 (1974-75), 82-105.

MilitAREV, A. Ju.: Istoričeskaja fonetika i leksika livijsko-guančskih jazykov. In: SolNCEV, V. M. (ed.): Jazyki Azii $i$ Afriki. IV, kniga 2. Moskva, 1991, Glavnaja Redakcija Vostočnoj Literatury. Pp. 238-267.

Monier-Williams, M.: A Sanskrit-English Dictionary. Etymologically and Philologically Arranged with Special Reference to Cognate Indo-European Languages. Delhi, 1899, Motilal Banarsidass Publishers.

Moreno, M. M.: Appunti sulla lingua darasa. In: Rendiconti della R. Accademia Nazionale dei Lincei. Classe di scienze morali, storiche e filologiche. Ser. VI, vol. XIII (1937), 211-240.

Moreno, M. M.: Manuale di sidamo. Grammatica, esercizi, testi, glossario. Roma, 1940, Casa Editrice A. Mondadori.

Mouchet, J.: Vocabulaires comparatifs de sept parlers du Nord-Cameroun. In: Bulletin de la Société d’Études Camerounaises 41-42 (1953), 136-206.

MukarovsKy, H. G.: Einige hamitosemitische und baskische Wortstämme. In: JungraithMAYR, H. \& MiEHE, G. (eds.): Berliner Afrikanistische Vorträge XXI. Deutscher Orientalistentag, Berlin 24.-29. März 1980. Berlin, 1981, Verlag von Dietrich Reimer. Pp. 105-118.

Mukarovsky, H. G.: Wo steht das Saharische? In: Afrika und Übersee 64 (1981), 187-226.

Mukarovsky, H. G.: Mande-Chadic Common Stock. A Study of Phonological and Lexical Evidence. Wien, 1987, Afro-Pub. NBÄ = OsING, J.: Die Nominalbildung des Ägyptischen. I-II. Maiz/Rhein, 1976, Verlag Philipp von Zabern.

NeTting, R. M.: Kofyar Vocabulary. MS. 1967.

NEWMAN, P.: The Formation of the Imperfective Verb Stem in Chadic. In: Afrika und Übersee 60/3 (1977), $178-192$.

NiCOLAs, F.: Vocabulaires ethnographiques de la Tamâjeq des Iullemmeden de l'est (Touâreg de la Colonie du Niger, Afrique Occidentale Française). In: Anthropos 52 (1957), 49-63, 564-580.

PARKer, E. M. \& HAYWARD, R. J.: An Afar-English-French Dictionary (with Grammatical Notes in English). London, 1985, School of Oriental and African Studies, University of London.

RdE $=$ Revue d'Égyptologie (Paris).

REINISCH, L.: Die Kafa-Sprache in Nordost-Afrika. II. Kafa-Deutsches Wörterbuch. In: Sitzungsberichte der Kaiserlichen Akademie der Wissenschaften. Phil.-hist. Classe 116 (1888), 251-386.

REINISCH, L.: Wörterbuch der Bedawye-Sprache. Wien, 1895, Alfred Hölder Verlag.

REINISCH, L.: Die Somali-Sprache. II. Wörterbuch. Wien, 1902, Alfred Hölder Verlag.

RENISIO, A.: Étude sur les dialectes berbères des Beni Iznassen, du Rif et des Senhaja de Sraïr. Grammaire, textes et lexique. Paris, 1932, Éditions Ernest Leroux.

ROPER, E.-M.: Tu Bedawic. An Elementary Handbook for the Use of Sudan Government Officials. Hertford, 1928, Stephen Austin \& Sons.

Rossing, M. O.: Mafa-Mada: A Comparative Study of Chadic Languages in North Cameroun. Ph.D. dissertation. Wisconsin, 1978, University of Wisconsin-Madison.

RÖSSLER, O.: Der semitische Charakter der libyschen Sprache. In: Zeitschrift für Assyriologie 50 (1952), 121-150.

RÖSSLER, O.: Libysch-Hamitisch-Semitisch. In: Oriens 17 (1964), 199-216.

SACHNINE, M.: Dictionnaire lamé-français. Lexique français-lamé. Paris, 1982, SELAF.

SASSE, H.-J.: Galla /š/, /s/ und /f/. In: Afrika und Übersee 58 (1975), 244-263.

SASSE, H.-J.: The Consonant Phonemes of Proto-East-Cushitic (PEC). In: Afroasiatic Linguistics 7/1 (1979), 1-67.

SASSE, H.-J.: An Etymological Dictionary of Burji. Hamburg, 1982, Helmut Buske Verlag.

SCHUH, R. G.: A Dictionary of Ngizim. Berkeley, California, 1981, University of California.

SED I = MilitareV, A. \& Kogan, L.: Semitic Etymological Dictionary. Volume One. Anatomy of Man and Animals. Münster, 2000, Ugarit-Verlag.

SHIMIZU, K.: The Southern Bauchi Group of Chadic Languages. A Survey Report. In: Africana Marburgensia. Special Issue 2 (1978), 1-50.

Siebert, R.: Languages of the Abbaya/Chamo Area - Report Part I (with Notes on Koorete by L. Hoeft). In: Survey of Little-Known Languages of Ethiopia (S.L.L.E.) Reports 21 (1994), 1-24.

Siebert, R. \& Siebert, K. \& WedeKInd, K.: Survey on Languages of the Asosa - Begi - Komosha Area. In: Survey of Little-Known Languages of Ethiopia (S.L.L.E.) Reports 11 (1993), 1-22. 
Siebert, R. \& WedeKIND, Ch.: Third S.L.L.E. Survey on Languages of the Begi/Asosa Area. In: Survey of LittleKnown Languages of Ethiopia (S.L.L.E.) Reports 15 (1994), 1-19.

SIRLINGER, E.: Dictionary of the Goemay Language. Jos, Nigeria, 1937, Prefecture Apostolic of Jos.

SKINNER, N.: Hausa Comparative Dictionary. Köln, 1996, Rüdiger Köppe Verlag.

Stolbova, O. V.: Opyt rekonstrukcii verhnezapadnočadskih kornej. In: Jazyki zarubežnogo Vostoka. Sbornik statej. Moskva, 1977, Nauka. Pp. 152-160.

Stolbova, O. V.: Studies in Chadic Comparative Phonology. Moscow, 1996, 'Diaphragma' Publishers.

STRÜMPELL, F.: Wörterverzeichnis der Heidensprachen des Mandara-Gebirges (Adamaua). In: Zeitschrift für Eingeborenen-Sprachen 13 (1922-23), 109-149.

TAKÁCS, G.: Refining Some Etymologies of the Root 'Round' in Afrasian and Egyptian (A Return to the Discussion in GL 34/1, 1994). In: General Linguistics 36/3 (1998), 153-166.

TAKÁCS, G.: Afro-Asiatic (Semito-Hamitic) Substratum in the Proto-Indo-European Cultural Lexicon? In: Lingua Posnaniensis 40 (1998), 141-172.

TAKÁCS, G.: Development of Afro-Asiatic (Semito-Hamitic) Compara-tive-Historical Linguistics in Russia and the Former Soviet Union. München, Newcastle, 1999, Lincom Europa.

TAKÁCS, G.: Contribution of V. M. Illič-Svityč to Chadic Comparative-Historical Linguistics. In: Archív Orientální 67 (1999), 361-378.

TAKÁCS, G.: Sibilant and Velar Consonants of South Cushitic and Their Regular Correspondences in Egyptian and Other Afro-Asiatic Branches. In: LAMBERTI, M. \& TONELli, L. (eds.): Afroasiatica Tergestina. Papers from the $9^{\text {th }}$ Italian Meeting of Afro-Asiatic (Hamito-Semitic) Linguistics, Trieste, April 23-24, 1998. Contributi presentati al 90 Incontro di Linguistica Afroasiatica (Camito-Semitica), Trieste, 23-24 Aprile 1998. Padova, 1999, Unipress. Pp. 393-426.

TAKÁCS, G.: South Cushitic Consonant System in Afro-Asiatic Context. In: Afrikanistische Arbeitspapiere 61 (2000), 69-117.

TAKÁCS, G.: The Afro-Asiatic Background of South Cushitic *h, ${ }^{*} h,{ }^{*} \uparrow,{ }^{*} h$, and ${ }^{*}$. In: Journal of Linguistic Relationship 4 (2010), 91-122.

TAKÁCS, G.: Some Berber Etymologies VIII: Berber Lexical Roots with *n- + labials. In: TOLLA, A.-M. di \& GHAKI, M. (éds.): Études berbères et libyco-berbères. Studi Africanistici. Napoli, 2011, l'Università degli Studi di Napoli 'L'Orientale'. Pp. 75-85.

TAKÁCS, G.: Some Berber Etymologies VII: Berber Lexical Roots with *n- + *-r/l/w/y-. In: MetTOUCHI, A. (éd.): «Parcours berbères». Mélanges offerts à Paulette Galand-Pernet et Lionel Galand pour leur 90 anniversaire. Köln, 2011, Rüdiger Köppe Verlag. Pp. 97-115.

Tourneux, H.: Une langue tchadique disparue: le muskum. In: Africana Marburgensia 10/2 (1977), 13-34.

Tourneux, H.: Le mulwi ou vulum de Mogroum (Tchad). Langue du groupe musgu - famille tchadique. Paris, 1978, Centre National de la Recherche Scientifique.

TOURneux, H.: Racine verbale en mulwi. In: CAPRILE, J.-P. \& JUnGRAITHMAYR, H. (eds.): Préalables à la reconstruction du proto-tchadique. Paris, 1978, SELAF. Pp. 89-93.

Tourneux, H. \& Seignobos, Ch. \& LAfarge, F.: Les Mbara et leur langue (Tchad). Paris, 1986, Société d'Études Linguistiques et Anthropologiques de France.

Tourneux, H.: Lexique pratique du munjuk des rizières. Dialecte de Pouss. Paris, 1991, Librairie Orientaliste Paul Geuthner.

Urk. II = SETHE, K.: Hieroglyphische Urkunden der griechisch-römischen Zeit, Leipzig, 1904, J. C. Hinrichs.

VYCICHL, W.: Is Egyptian a Semitic Language? In: Kush 7 (1959), 27-44.

VyCICHL, W.: Studien der ägyptisch-semitischen Wortvergleichung. Die Klassifikation der Etymologien. Zwölf neue Etymologien. In: Zeitschrift für Ägyptische Sprache 84 (1959), 70-74.

WÄDN = DEINES, H. von \& GRAPOW, H.: Wörterbuch der ägyptischen Drogennamen. Berlin, 1959, AkademieVerlag.

Wb = ERMAN, A. \& GRAPOW, H.: Wörterbuch der ägyptischen Sprache. I-V. ${ }^{2}$ Berlin, 1957-1971, Akademie-Verlag.

Wedekind, K.: Gimo-Jan or Ben-Yem-Om: Benč-Yemsa Phonemes, Tones, and Words. In: HAYWARD, R. (ed.): Omotic Language Studies. London, 1990, SOAS. Pp. 68-141.

WUS = AISTLEITNER, J.: Wörterbuch der ugaritischen Sprache. Berichte über die Verhandlungen der Sächsischen Akademie der Wissenschaften zu Leipzig. Phil.-hist. Klasse 106/3 (1963).

ZDMG = Zeitschrift der Deutschen Morgenländischen Gesellschaft (Wiesbaden). 
Статья является очередной публикацией в специальной серии, посвященной исследованию исконно афразийского слоя в лексике омотских языков, распределенной по начальным согласным корня. В настоящей работе исследуются омотские лексемы на * $p$ - и ${ }^{*} p^{h_{-}}$, которые могут быть возведены к соответствующим праафразийским корням на * $p$ и *f-. Всего в статье обсуждается 28 этимологий, удовлетворяющих этим условиям.

Ключевые слова: афразийские языки, омотские языки, сравнительно-историческая фонетика, лингвистическая реконструкция. 